\title{
LAND SNAILS (MOLLUSCA: GASTROPODA)OF ISLANDS ŽIRJE, KAPRIJE AND PRVIĆ (CROATIA)
}

\section{Vesna Štamol, Eduard KletečKi \& Marijana Vuković}

\author{
Croatian Natural History Museum, Demetrova 1, 10000 Zagreb, Croatia
}

Štamol, V., Kletečki, E. \& Vuković, M.: Land snails (Mollusca: Gastropoda) of islands Žirje, Kaprije and Prvić (Croatia). Nat. Croat., Vol. 27, No. 1, 195-209, Zagreb, 2018.

Land malacofauna of three islands of the Šibenik archipelago (Dalmatia, Croatia), Žirje, Kaprije and Prvić was studied. During field work, the highest number of species, 31, was recorded on the largest island, Žirje; on the mid-sized island of Kaprije, there were 27 species, and on the smallest of them, the island of Prvić, there were 20 species. We confirmed all the species recorded previously in literature, six for Žirje and one for Prvić. In the fauna we emphasize the recently described species Hypnophila zirjensis Štamol, Manganelli, Barbato \& Giusti, 2018, so far found only on Žirje, Truncatellina lussinensis Štamol, 1995 was found on Kaprije which is the second find after its locus typicus on Lošinj island, and Delima (Semirugata) vidovichii vidovichii (L. Pfeiffer, 1846) on Prvić, which is the first island record of this taxon. Comparison of these malacofaunas with Adriatic islands similar in size - Žirje with Silba and Prvić with Piškera, showed that Žirje and Prvić have more species, which we assigned to the more diverse habitats.

Key words: land snails, Adriatic islands, Žirje, Kaprije, Prvić

Štamol, V., Kletečki, E. \& Vuković, M.: Kopneni puževi (Mollusca: Gastropoda) otoka Žirja, Kaprija i Prvića (Hrvatska). Nat. Croat., Vol. 27, No. 1, 195-209, Zagreb, 2018.

Obrađena je kopnena malakofauna triju otoka Šibenskog arhipelaga (Dalmacija, Hrvatska), Žirja, Kaprija i Prvića. Terenskim istraživanjem najviše je vrsta nađeno na najvećem otoku, Žirju - 31 vrsta, na srednje velikom otoku Kapriju nađeno je 27 vrsta, a najmanje - 20 vrsta, na najmanjem otoku, Prviću. Potvrdili smo postojanje svih literaturno zabilježenih vrsta, 6 za Žirje i jedne za Prvić. U fauni se ističu nedavno opisana vrsta Hypnophila zirjensis Štamol, Manganelli, Barbato \& Giusti, 2018, dosada nađena samo na Žirju, Truncatellina lussinensis Štamol, 1995 nađena na Kapriju, što je prvi nalaz nakon locusa typicusa na Lošinju, te na Prviću Delima (Semirugata) vidovichii vidovichii (L. Pfeiffer, 1846) što je njen prvi otočni nalaz. Usporedba s malakofaunom slično velikih jadranskih otoka - Žirja sa Silbom te Prvića s Piškerom, pokazala je da Žirje i Prvić imaju više vrsta, što smo pripisali raznolikijim staništima.

Ključne riječi: kopneni puževi, jadranski otoci, Žirje, Kaprije, Prvić

\section{INTRODUCTION}

With this paper we continue the publication of results of research of Croatian Adriatic islands with unknown or poorly known land malacofauna. The first author has already elaborated, with or without co-authorship, a few Croatian north Adriatic islands belonging to the Kvarner area - Cres, Lošinj (ŚtAmol \& Velkovrh, 1995) and Susak (ŠTAMOL \& PoJE, 1998), as well as some islands situated more to the south and belonging to Dalmatia - the north Dalmatian island of Dugi otok (ŠTAMOL, 2004; ŠTAMOL \& KLETEČKI, 2005), Kornat and the islands of the Kornati NP (ŠтAмоL et al., 2012), and the central Dalmatian islands of Šolta, Drvenik veli, Drvenik mali (ŠTAmol et al., 2017) and Brač (Š́tAMOL, 1986). In this work we aimed to study land snails of three further islands of 
northern Dalmatia, belonging to the Šibenik island group, Žirje, Kaprije and Prvić. The research was also motivated by the recent discovery of the new species on Žirje, Hypnophila zirjensis Štamol, Manganelli, Barbato \& Giusti, 2018 (Šтамо et al., 2018).

\section{STUDY AREA}

The studied islands of Žirje, Kaprije and Prvić are situated in northern Dalmatia (Croatia) (Fig. 1). The closest relatively large city is Šibenik, and so they belong to the Šibenik archipelago. All islands lie in the Dinaric direction characteristic of the entire Adriatic coast (from NW towards SE). Climate is typically Mediterranean with mild, rainy winters and hot, dry summers. In those conditions eu-Mediterranean vegetation has developed, represented by stony grasslands and macchias of the alliance Quercion ilicis, while forests of Aleppo pine have been planted as well (Franjıć \& PANDŽA, 1996; PANDŽA, 2003; MiLOVIĆ \& PANDŽA, 2010).
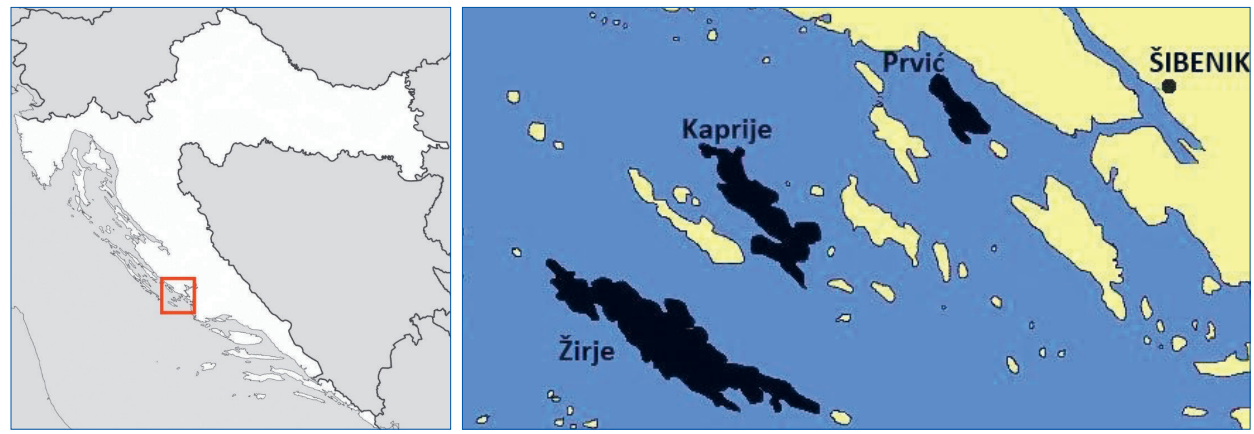

Fig. 1. Position of the researched islands in Croatia.

The islands differ in, among other things, size, distance from mainland and human presence. The biggest of them, the offshore island of Žirje, is the most distant and the least populous. It has an area of $15.08 \mathrm{~km}^{2}$ (DuPLANČIĆ Leder et al., 2004). It is $12 \mathrm{~km}$ long and on average $1.2 \mathrm{~km}$ wide (Friganović, 1994). Its highest summit is Kapić, $134 \mathrm{~m}$ a.s.l (BognAR \& Saletto-Janković, 1994) and it lies $11.8 \mathrm{~km}$ from the mainland. According to the 2011 population census there are only 103 inhabitants (BuRšı́c, 2013). In a geological framework, Upper Cretaceous limestones dominate, extending in the NW-SE direction (BoGNAR \& Saletto-Janković, 1994). The valley located in the middle of the island is covered with a thin layer of red soil overlaid with sediments and turned brown (BoGNAR \& SALETTO-JANKOvić, 1994). Speleological objects on the island are in the form of pits (BARIšić, 1994; JALŽIĆ, 1994). The average annual temperature is $15.6^{\circ} \mathrm{C}$, and average annual precipitation 753 $\mathrm{mm}$. Relative annual humidity of $78 \%$, i.e. $64 \%$ in summer, along with dew, compensate for the small amount of precipitation (Friganović, 1994). Agriculture is poorly developed, although among all Šibenik islands, Žirje has the biggest and most valuable arable land areas (FARIČIĆ \& MAGAš, 2004). In the fields people grow the vine, figs, olives, plums and vegetables (PANDžA, 2003). The island is characterized by depopulation and agriculture abadonment, which leads the arable land being overgrown.

Of these three islands, Kaprije is the middle in terms of size, distance from the mainland $(8.5 \mathrm{~km})$ and population. It has an area of $7.12 \mathrm{~km}^{2}$ (DUPLANČić LeDER et al., 2004), and its height is $129 \mathrm{~m}$ (FrAnjIĆ \& PANDŽA, 1996). The island is built of limestones with 
dolomites (FrANJić \& PANDŽA, 1996). There is no meteorogical station there so we use weather data for the wider Šibenik area, in which there is an average annual temperature of $15.4{ }^{\circ} \mathrm{C}$ and an average annual precipitation of $690 \mathrm{~mm}$ (PANDžA, 1998). According to the 2011 population census there are 189 inhabitants on the island (BuRšıć, 2013). Agricultural land is mainly abandoned, there are only gardens in the settlement and a few olive groves in its surroundings (FranjIć \& PANDŽA, 1996).

The island of Prvić is the smallest $\left(2.41 \mathrm{~km}^{2}\right)$ (Duplančić Leder et al., 2004) and the closest to the mainland $(0.85 \mathrm{~km})$. It is probably this last fact that makes this island the most populated: it has 403 inhabitants according to the 2011 population census (Buršić, 2013). The highest summit is $79 \mathrm{~m}$ a.s.l. Prvić is built of dolomites and calcites (PANDŽA, 1998). As on Kaprije, on Prvić there is no meteorogical station and we also use weather data for the wider Šibenik area. Regarding cultivated land on the island there are mainly gardens, and abandoned fields turned into grasslands (PANDŽA, 1998). Unfortunately, due to developing tourism, and due to the proposed building of a marina that local people are trying to prevent, there is a threat of devastation to the remaining non-urbanized areas.

\section{The history of the research}

According to the available literature the researched islands have been either not researched at all, or only poorly and unsistematically. For example for the island of Kaprije there is not a single data about its malacofauna. For the island of Prvić, thanks to the Italian naturalist Cristophoro Bellotti who in 1853 travelled through Dalmatia, and another Italian naturalist, Pelegrino Strobel, who studied the land snails collected then, only one piece of information has been published, and about only one species (STROBEL, 1854). For the island of Žirje there are three papers listing its land snails, in the oldest among them one species (BRUSINA, 1872), in the second of them 3 species (FrANK, 1991), and in the most recent two species (ŠTAmol et al., 2018). The oldest one is a result of a casual observation of a land snail by the versatile Croatian naturalist, primarily malacologist, Spiridion Brusina, while he was researching marine flora and fauna on Žirje in 1868 (BrusinA, 1872). Data from the second paper are result of random collection by the Austrian entomologist Wolfgang Suppantschitsch in 1984 (FRANK, 1991). In the recently published paper, dedicated to the description of a new taxon, two species are mentioned (ŠTAmol et al., 2018). This is also the only paper with more detailed island finding sites. Altogether only 6 species of land snails were recorded for Žirje. Based on everything mentioned above, we can conclude that the land malacofauna of these islands has so far been unknown (Kaprije), or very poorly known (Prvić and Žirje).

\section{MATERIALS AND METHODS}

Field research into the terrestrial malacofauna of islands Žirje and Kaprije was conducted at the end of October and in the beginning of November 2004, and into that of the island of Prvić on June 3rd, 2008. Larger land snail species were collected individually, and for smaller species soil samples were collected, from which shells were isolated after drying and sifting through sieves of decreasing mesh size. Each locality was browsed by two re-

\footnotetext{
* In Croatia there are two islands named Prvić; one in the northern Adriatic near the city of Senj, and the other in Dalmatia near the city of Šibenik. Data from STrobel (1854) can without a doubt be assigned to the latter one.
} 
searchers for about 30 minutes. Because of the short stay on Prvić, at three localities (loc. 44,45 and 47), only randomly observed large species were noted. Localities were arranged in a way to cover all main habitats and to form a vertical and a horizontal grid of the island. Terrestrial fauna was collected by the employees of the Croatian Natural History Museum (Zagreb, Croatia), curators Vesna Štamol and Eduard Kletečki on Kaprije and Žirje, and Vesna Štamol and senior museum taxidermist Zlatko Godec on Prvić. The material from the Gradina pit on Žirje was collected by biospeleologist, retired senior museum technician Branko Jalžić in 2004 and 2007, and this is the only malacologically investigated speleological object. Slugs were not a part of this research because of the different methods required for collecting them. Snails were identified based on their shells. The material is deposited in the General Collection of Recent Mollusca of the Croatian Natural History Museum (Zagreb, Croatia).

\section{A list of sites}

In the list we give researched localities, and in the brackets there are toponym type (e.g. summit, hamlet, bay), altitude, UTM designation, GPS coordinates and habitat. GPS coordinates were not taken using a GPS device during the field survey, but were subsequently determined from the 1:25000 topographic maps of the Military GeoGRAPHY INSTITUTE (1984), and are given in the WGS84 coordinate system. Toponyms were taken also from the mentioned topographic maps 1:25000. Locality numbers 1-25 apply to Žirje, 26-41 to Kaprije, and 42-50 to Prvić. Positions of localities are shown by associated ordinal numbers on Fig. 2A-C.
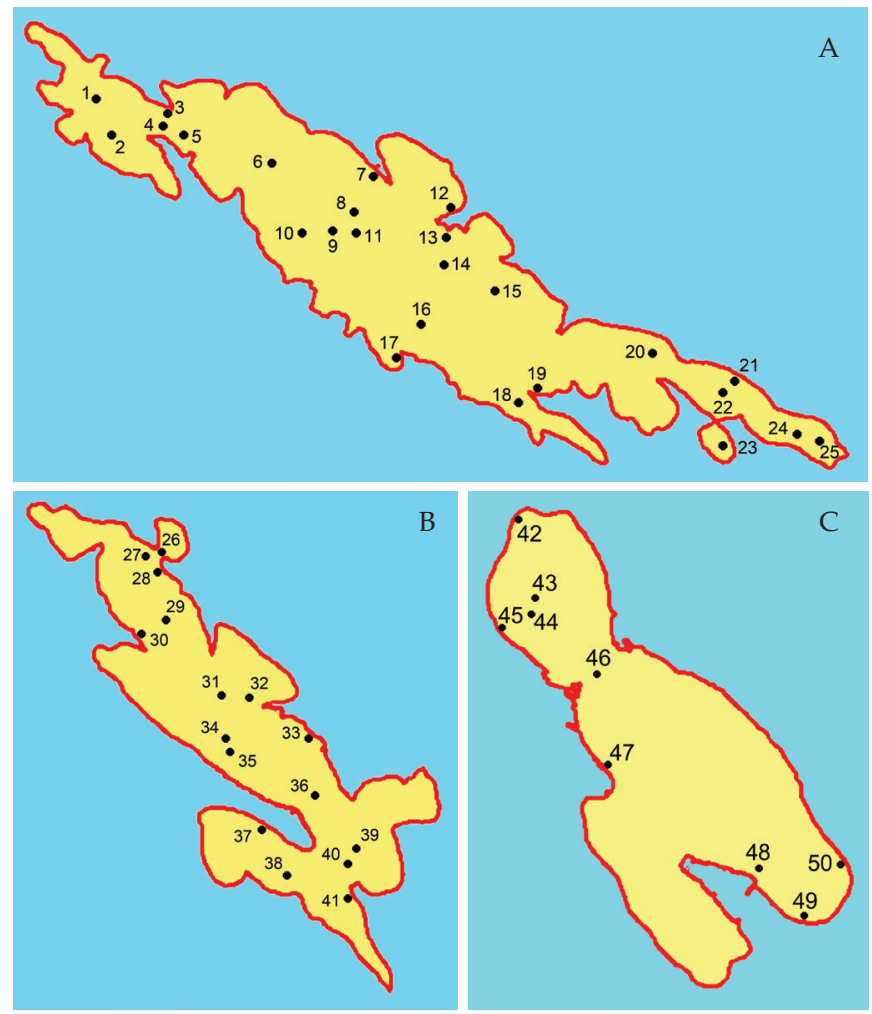

Fig. 2A-C. Position of study localities on Žirje (A), Kaprije (B) and Prvić (C) islands. Numbers in the figure correspond to the numbers in the list of sites. 
1. Žirje (island), Jajni vrsi (summits); $80 \mathrm{~m}$ a.s.1.; WJ43; $43.6703^{\circ} \mathrm{N}, 15.6115^{\circ} \mathrm{E}$; stony grassland, rocks.

2. Žirje (island), Vela glava (summit); $90-103 \mathrm{~m}$ a.s.l.; WJ43; $43.6657^{\circ} \mathrm{N}$, $15.6142^{\circ} \mathrm{E}$; stony grassland, rocks.

3. Žirje (island), Sv. Nikola (church), Mikavica (bay); $5 \mathrm{~m}$ a.s.1.; WJ53; $43.6684^{\circ} \mathrm{N}, 15.6234^{\circ} \mathrm{E}$; grassland, rocks, along walls.

4. Žirje (island), Tratinska (bay); $5 \mathrm{~m}$ a.s.1.; WJ53; $43.6666^{\circ} \mathrm{N}, 15.6226^{\circ} \mathrm{E}$; near stone house.

5. Žirje (island), Tratinska (bay); $20 \mathrm{~m}$ a.s.l.; WJ53; $43.6655^{\circ} \mathrm{N}, 15.6260^{\circ} \mathrm{E}$; stony grassland, rocks.

6. Žirje (island), Polje (region) - N part; $20 \mathrm{~m}$ a.s.1.; WJ53; $43.6629^{\circ} \mathrm{N}$, $15.6390^{\circ} \mathrm{E}$; olive grove, dry stone walls.

7. Žirje (island), Muna (hamlet); 3-15 $\mathrm{m}$ a.s.1.; WJ53; $43.6617^{\circ} \mathrm{N}, 15.6556^{\circ} \mathrm{E}$; dry stone walls, house walls, rocks.

8. Žirje (island), Žirje (village); $20-30 \mathrm{~m}$ a.s.1.; WJ53; $43.6574^{\circ} \mathrm{N}, 15.6517^{\circ} \mathrm{E}$; house walls.

9. Žirje (island), Polje (region) - middle part, at pool; $10 \mathrm{~m}$ a.s.l.; WJ53; $43.6553^{\circ} \mathrm{N}, 15.6490^{\circ} \mathrm{E}$; dry stone walls.

10. Žirje (island), Straža (summit); $110 \mathrm{~m}$ a.s.l.; WJ53; $43.6543^{\circ} \mathrm{N}, 15.6445^{\circ} \mathrm{E}$; stony grassland, rocks, under ivy.

11. Žirje (island), Žirje (village); $18-20 \mathrm{~m}$ a.s.1.; WJ53; $43.6552^{\circ} \mathrm{N}, 15.6533^{\circ} \mathrm{E}$; stone house, dry stone walls.

12. Žirje (island), Koromašna (bay)-N part; $5-20 \mathrm{~m}$ a.s.1.; WJ53; $43.6577^{\circ} \mathrm{N}$, $15.6693^{\circ} \mathrm{E}$; rocks.

13. Žirje (island), Koromašna (bay)-S part; $5-15 \mathrm{~m}$ a.s.1.; WJ53; $43.6548^{\circ} \mathrm{N}$, $15.6672^{\circ} \mathrm{E}$; gardens, rocks.

14. Žirje (island), Mikuljica (region); $70 \mathrm{~m}$ a.s.l.; WJ53; $43.6517^{\circ} \mathrm{N}, 15.6666^{\circ} \mathrm{E}$; grassland, dry stone walls.

15a. Žirje (island), Kapić (summit); 130-134 m a.s.1.; WJ53; $43.6475^{\circ} \mathrm{N}$, $15.6750^{\circ} \mathrm{E}$; rocks.

15b. Žirje (island), Kapić (summit); 100-110 m a.s.1.; WJ53; $43.6468^{\circ} \mathrm{N}$, $15.6741^{\circ} \mathrm{E}$; rocks.

16. Žirje (island), Polje (region) - S part; $20 \mathrm{~m}$ a.s.1.; WJ53; $43.6441^{\circ} \mathrm{N}$, $15.6632^{\circ} \mathrm{E}$; stone house, dry stone wall, olive grove.

17. Žirje (island), Kruševica (bay); 5-10 $\mathrm{m}$ a.s.1.; WJ53; $43.64034^{\circ} \mathrm{N}, 15.6601^{\circ} \mathrm{E}$; rocks, garrigue, pine grove.

18. Žirje (island), Gradina (region) (Fig. 4); $30 \mathrm{~m}$ a.s.1.; WJ53; $43.6321^{\circ} \mathrm{N}$, $15.6817^{\circ} \mathrm{E}$; dry stone walls, rocks, pit.

19. Žirje (island), Stupica (hamlet); $2-5 \mathrm{~m}$ a.s.1.; WJ53; $43.6368^{\circ} \mathrm{N}, 15.6809^{\circ} \mathrm{E}$; dry stone walls, garden, pine grove.

20. Žirje (island), Gušterne (hill); 75-81 $\mathrm{m}$ a.s.1.; WJ53; $43.6409^{\circ} \mathrm{N}, 15.7001^{\circ} \mathrm{E}$; stony grassland, rocks.

21. Žirje (island), Zvizdulja (summit); $15 \mathrm{~m}$ a.s.l.; WJ53; $43.6388^{\circ} \mathrm{N}, 15.7121^{\circ} \mathrm{E}$; grassland, house wall;

22. Žirje (island), Zvizdulja (summit); $85-91 \mathrm{~m}$ a.s.1.; WJ53; $43.6368^{\circ} \mathrm{N}$, $15.7117^{\circ} \mathrm{E}$; rocks; 
23. Žirje (island), Kabal (summit); 55-61 $\mathrm{m}$ a.s.1.; WJ53; $43.6304^{\circ} \mathrm{N}, 15.7104^{\circ} \mathrm{E}$; stony ground, rocks.

24. Žirje (island), Smrikovac (summit); $40 \mathrm{~m}$ a.s.1.; WJ53; $43.6328^{\circ} \mathrm{N}, 15.7225^{\circ} \mathrm{E}$; stony grassland, rocks.

25. Žirje (island), Smrikovac (summit); $70-80 \mathrm{~m}$ a.s.1.; WJ53; $43.6319^{\circ} \mathrm{N}$, $15.7253^{\circ} \mathrm{E}$; rocks.

26. Kaprije (island), Medoš (hamlet); $2-3 \mathrm{~m}$ a.s.1.; WJ54; $43.7146^{\circ} \mathrm{N}, 15.6892^{\circ} \mathrm{E}$; near houses, dry stone walls.

27. Kaprije (island), Medoš (hamlet) - Mali Uljenak (summit); 20 m a.s.l.; WJ54; $43.7141^{\circ} \mathrm{N}, 15.6871^{\circ} \mathrm{E}$; under stones, dry stone walls.

28. Kaprije (island), Medoš (hamlet), Zmorašnji Medoš (bay); 2-10 m a.s.l.; WJ54; $43.7128^{\circ} \mathrm{N}, 15.6894^{\circ} \mathrm{E}$; stony grassland, dry stone walls.

29. Kaprije (island), Veli Uljenak (summit); $115-121 \mathrm{~m}$ a.s.1.; WJ54; $43.7066^{\circ} \mathrm{N}$, $15.6910^{\circ} \mathrm{E}$; rocks, dry stone walls, macchia.

30. Kaprije (island), Divarka (region), Vanjska uvala (bay); 20 m a.s.l.; WJ54; $43.7055^{\circ} \mathrm{N}, 15.6870^{\circ} \mathrm{E}$; rocks.

31. Kaprije (island), Škraljica (region); $40 \mathrm{~m}$ a.s.1.; WJ53; $43.7012^{\circ} \mathrm{N}, 15.6979^{\circ} \mathrm{E}$; stony grassland, dry stone walls.

32. Kaprije (island), Luka (bay) (Fig. 5); $40 \mathrm{~m}$ a.s.1.; WJ53; $43.7000^{\circ} \mathrm{N}, 15.7024^{\circ} \mathrm{E}$; rocks.

33. Kaprije (island), Mulo (bay); 2-10 m a.s.l.; WJ53; $43.6956^{\circ} \mathrm{N}, 15.7114^{\circ} \mathrm{E}$; rocks, pine grove, olive grove.

34. Kaprije (island), Prvi vrh (summit); $80 \mathrm{~m}$ a.s.l.; WJ53; $43.6940^{\circ} \mathrm{N}, 15.7000^{\circ} \mathrm{E}$; rocks.

35. Kaprije (island), Babin vrh (summit); $50 \mathrm{~m}$ a.s.1.; WJ53; $43.6930^{\circ} \mathrm{N}$, $15.7008^{\circ} \mathrm{E}$; dry stone walls.

36. Kaprije (island), Kaprije (village); 20-40 m a.s.1.; WJ53; $43.6902^{\circ} \mathrm{N}$, $15.7113^{\circ} \mathrm{E}$; dry stone walls, house walls, olive grove, gardens.

36a. Kaprije (island), Kaprije (village); $30 \mathrm{~m}$ a.s.1.; WJ53; $43.6894^{\circ} \mathrm{N}, 15.7131^{\circ} \mathrm{E}$; church, cemetery, dry stone walls, monument in the port.

37. Kaprije (island), Luka Kaprije (bay) - W part; 2-10 m a.s.l.; WJ53; $43.6862^{\circ} \mathrm{N}, 15.7047^{\circ} \mathrm{E}$; rocks, dry stone walls, olive grove, garden.

38. Kaprije (island), Glavičica (summit); $60 \mathrm{~m}$ a.s.1.; WJ53; $43.6815^{\circ} \mathrm{N}$, $15.7087^{\circ} \mathrm{E}$; rocks, pine grove.

39. Kaprije (island), Vela glava (summit); $110-129 \mathrm{~m}$ a.s.l.; WJ53; $43.6846^{\circ} \mathrm{N}$, $15.7174^{\circ} \mathrm{E}$; stony ground, stony grassland.

40. Kaprije (island), Vela glava (summit); 60-70 $\mathrm{m}$ a.s.1.; WJ53; $43.6830^{\circ} \mathrm{N}$, $15.7157^{\circ} \mathrm{E}$; rocks.

41. Kaprije (island), Nozdra mala (bay) (Fig. 5); $2-5 \mathrm{~m}$ a.s.1.; WJ53; $43.6799^{\circ} \mathrm{N}$, $15.7167^{\circ} \mathrm{E}$; rocks, pine grove.

42. Prvić (island), Rt Sv. Luce (cape); $5 \mathrm{~m}$ a.s.l.; WJ64; $43.7430^{\circ} \mathrm{N}, 15.7830^{\circ} \mathrm{E}$; pine grove, garrigue, stony grassland.

43. Prvić (island), Grbelja (region); $45 \mathrm{~m}$ a.s.l.; WJ64; $43.7394^{\circ} \mathrm{N}, 15.7842^{\circ} \mathrm{E}$; near old houses walls, stony grassland.

44. Prvić (island), Grbelja (region); $30 \mathrm{~m}$ a.s.1.; WJ64; $43.7381^{\circ} \mathrm{N}, 15.7834^{\circ} \mathrm{E}$; dry stone walls. 
45. Prvić (island), Rt Svete Luce (cape) - Prvić Šepurine (village); $5 \mathrm{~m}$ a.s.l.; WJ64; $43.7377^{\circ} \mathrm{N}, 15.7813^{\circ} \mathrm{E}$; dry stone walls.

46. Prvić (island), Prvić Šepurine (village); $11 \mathrm{~m}$ a.s.1.; WJ64; $43.7345^{\circ} \mathrm{N}$, $15.7895^{\circ} \mathrm{E}$; grassland, by the walls.

47. Prvić (island), Trstevica (bay); $1-2 \mathrm{~m}$ a.s.1.; WJ64; $43.7294^{\circ} \mathrm{N}, 15.7901^{\circ} \mathrm{E}$; abandoned gardens.

48. Prvić (island), Prvić Luka (village); $3 \mathrm{~m}$ a.s.1.; WJ64; $43.7247^{\circ} \mathrm{N}, 15.8004^{\circ} \mathrm{E}$; cemetery.

49. Prvić (island), Sv. Ante (chapel); $2-3 \mathrm{~m}$ a.s.1.; WJ64; $43.7221^{\circ} \mathrm{N}, 15.8046^{\circ} \mathrm{E}$; dry stone walls.

50. Prvić (island), Lovrenčevica (cape); $4 \mathrm{~m}$ a.s.l.; WJ64; $43.7247^{\circ} \mathrm{N}, 15.8066^{\circ} \mathrm{E}$; dry stone walls, stony grassland.

\section{RESULTS AND DISCUSSION}

\section{List of terrestrial snails with finding sites}

In the list we give species and subspecies of land snails found during our field work and recorded in the literature. Nomenclature is given and taxa are listed in order according to ŠTAMOL (2010). In front of the valid taxon name in most cases there is an ordinal number. The absence of an ordinal number denotes specimens that in certain localities could not be identified to species level - mostly because they were juvenile or badly preserved, although members of the same genus could be identified in other localities. After the designation "N:" we cite the taxon name used in the paper mentioning the finding sites on the researched islands. Field work sites are given after the designation " $F$ " with their number from the list of sites and Figs 2A-C. Field work sites for Zirje are marked in black, for Kaprije red, and for Prvić in blue numbers. A question mark by the site number denotes that specimens from that site could not be assigned with certainty to the mentioned taxon. Finding sites from literature are given after the designation "L:".

\section{Cochlostoma (Cochlostoma) scalarinum scalarinum (A. \& B. J. Villa, 1841)}

N: Cochlostoma (Cochlostoma) scalarinum Villa 1841: FrANK, 1991: 351.

F: 1, 2, 3, 4, 5, 6, 7, 8, 9, 10, 11, 12, 13, 14, 15, 16, 17, 18, 19, 20, 22, 23, 24, 25, 26, 27, $28,29,30,31,32,33,34,35,36 a, 37,38,39,40,41,50$.

L: Žirje island: „I. Žirje“ (FrANK, 1991: 351).

Remark: The latest genus revision (ZALlot et al., 2015) placed C. scalarinum into Wagneriola subgenus.

\section{Pomatias elegans (O. F. Müller, 1774)}

F: 1, 3, 4, 5, 6, 7, 8, 9, 11, 13, 20, 21, 22, 25, 26, 27, 28, 30, 31, 32, 36, 36a, 38, 42, 43, 46, 48, 49, 50.

3. Cecilioides (Cecilioides) acicula (O. F. Müller, 1774)

F: 4, 7, 12, 21, 28, 32?, 34, 39, 40.

4. Cecilioides (Cecilioides) veneta (Strobel, 1855)

F: 2?, 3, 11?, 12, 13, 16, 21, 28, 32?, 34, 41. 


\section{Cecilioides sp.}

F: $6,9,26,38,43$.

5. Rumina decollata (Linnaeus, 1758)

F: 36 .

6. Charpentieria (Gibbularia) gibbula gibbula (Rossmässler, 1836)

F: 7, 8, 11, 42, 43, 46, 49 .

7. Delima (Delima) blanda conspurcata (Rossmässler, 1836)

F: 3, 8, 9, 11, 36, 36a.

8. Delima (Semirugata) semirugata semirugata (Rossmässler, 1836)

N: Delima semirugata (Rossmaessler 1836): FrAnK, 1991: 362;

F: $3,4,5,6,11,12,13,14,15,16,18,19,20,21,24,26,27,28,30,33,34,35,36,36$, 40, 41, 50 .

L: Žirje island: „I. Žirje“ (FRANK, 1991: 362).

9. Delima (Semirugata) vidovichii robusta (Küster, 1847)

F: 7, 22, 31, 36, 36a, 37, 39, 40, 41.

10. Delima (Semirugata) vidovichii vidovichii (L. Pfeiffer, 1846)

F: 43,48 .

Delima sp.

F: 2, 29, 43, 49 .

11. Hypnophila pupaeformis (Cantraine, 1836)

N: Hypnophila pupaeformis (Cantraine, 1835): ŠтамоL et al., 2018: 89;

F: $3,4,7,8,9,11,13,15,16,18,20,21,22,26,27,28,29,30,31,32,33,34,35,36$, $36 a, 37,38,40,41,46,48,49,50$.

L: Žirje island: „Žirje Island“ (ŠтAмol et al., 2018: 89).

12. Hypnophila zirjensis Štamol, Manganelli, Barbato \& Giusti, 2018 (Fig. 3)

N: Hypnophila zirjensis n. sp.: ŠтAмоL et al., 2018

F: 1, 2, 12, 17, 18, 19, 22, 25.

L: Žirje island: „Gradina (pit), Gradina (area)“ (ŠTAmol et al., 2018: 93); „Jajni vrsi (summit)" (Šтамоl et al., 2018: 93); „Kruševica (bay)“ (Šтамоl et al., 2018: 93); „Smrikovac (summit), Stupica (village)“ (ŠтAмol et al., 2018: 93); „Vela glava (summit)“ (SтAмоl et al., 2018: 93); "Zvizdulja (summit)“ (SтAмоl et al., 2018: 93).

13. Pseudochondrula seductilis seductilis (Rossmässler, 1837)

F: $3 ?, 4$.

14. Mediterranea hydatina hydatina (Rossmässler, 1838)

F: $11,36,43,46,48,49,50$.

15. Oxychilus sensu lato sp.

F: 9, 22, 25, 40, 42.

16. Vitrea botterii (L. Pfeiffer, 1853)

F: $9,10,12,22,26,28,31,32,33,34,35,36,39,40,41$.

17. Vitrea subrimata (Reinhardt, 1871)

F: 2, 3, 4, 6, 8, 10, 11, 12, 13, 15, 16, 19, 20, 24, 27, 28, 29, 30, 34, 38, 39, 40, 41, 42.

Vitrea sp.

F: 14, 25. 
18. Chilostoma (Liburnica) setosa setosa (A. Férussac, 1832)

F: 46.

19. Cornu aspersum aspersum (O. F. Müller, 1774)

F: 5, 7, 8, 9, 11, 13, 19, 21, 33, 36, 36a, 44, 45, 46, 48, 49.

20. Eobania vermiculata vermiculata (O. F. Müller, 1774)

N: Helix vermiculata Müll.: STRobel, 1854: 122.

F: $2,4,5,6,7,8,9,11,12,13,16,19,20,21,26,28,31,32,33,35,36,36 a, 37,38,40$, 41, 42, 43, 44, 46, 48, 49, 50.

L: Prvić island: „Provicchio“ (Strobel, 1854: 122 ).

21. Helix (Helix) cincta cincta O. F. Müller, 1774

N: Helix (Pomatia) cincta Müll.: Brusina, 1872: 131.

F: 3, 6, 8, 9, 11, 14, 19, 28, 36, 36a, 47.

L: Žirje island: „otok Zuri“ (BrusinA, 1872: 131).

22. Cernuella (Cernuella) cisalpina cisalpina (Rossmässler, 1837)

F: $46,48,49$.

23. Cernuella (Cernuella) virgata (Da Costa, 1778)

F: 6, 7, 8, 14, 21, 25, 28, 36.

Cernuella (Cernuella) sp.

F: 9, 19, 36a.

24. Monacha (Monacha) cartusiana (O. F. Müller, 1774)

F: $3,4,6,7,8,9,10,11,19,26,27,28,29,31,32,33,34,35,36,36 a, 37,38,39,40$, $41,42,43,46,49,50$.

25. Monacha (Monacha) parumcincta (Menke, 1828)

F: 1, 2, 3, 4, 5, 7, 10, 12, 13, 14, 15, 16, 17, 18, 19, 20, 21, 22, 23, 24, 25.

Monacha sp.

F: 30,48 .

27. Punctum (Punctum) pygmaeum (Draparnaud, 1801)

F: 19, 33, 38, 39, 41.

28. Granaria illyrica illyrica (Rossmässler, 1835)

N: Granaria illyrica (Rossmaessler 1837): FRANK, 1991: 355.

F: 1, 2, 4, 12, 13, 15, 18, 19, 20, 22, 23, 24, 25, 29, 30, 32, 33, 34, 35, 36, 38, 39, 40, 41.

L: Žirje island: „Insel Žirje“ (FrANK, 1991: 355).

Remark: According to FeHÉr et al. (2010) G. illyrica is a subspecies of G. frumentum (Draparnaud, 1801).

29. Granopupa granum (Draparnaud, 1801)

F: $12,19$.

30. Rupestrella philippii philippii (Cantraine, 1840)

F: $2,7,30$.

31. Rupestrella rhodia (Roth, 1839)

F: $8,11,12,13,15,16,18,20,25,26,30,32,34,35,36,38,39,40,41$.

32. Lauria (Lauria) cylindracea (Da Costa, 1778)

F: 3, 4, 7, 8, 9, 10, 11, 14, 16, 26, 33, 36, 36a, 42, 46.

33. Pyramidula sp.

F: $3,4,6,8,10,11,14,15,16,20,26,37,43$. 


\section{Acanthinula aculeata (O. F. Müller, 1774)}

F: 39 .

35. Truncatellina callicratis (Scacchi, 1833)

F: 4, 7, 13, 14, 16, 19, 26, 28, 29, 33, 34, 36a, 37, 39, 42, 43.

36. Truncatellina lussinensis Štamol, 1995

\section{F: 26.}

\section{Truncatellina sp.}

F: 9, 11.

\section{Poiretia cornea (Brumati, 1838)}

F: $1,2,3,4,6,7,8,9,10,11,13,15,16,18,19,20,21,22,25,26,27,28,29,30,31$, $32,33,34,35,36,36 a, 37,38,39,40,41,43,46,48,49,50$.

\section{Aegopis acies (A. Férussac, 1832)}

F: $44,48,49,50$.

Snails collected during field work were identified to the smallest taxonomical category enabled by the shell morphology. For most taxa this is species and/or subspecies level. The majority of the genus Pyramidula could not be differentiated by shell (RAzKIN et al., 2017), and it is possible that on the researched islands this genus is represented by more species. The case with Oxychilus sensu lato is similar. Therefore we can conclude that during field work on the islands of Žirje, Kaprije and Prvić at least 38 species of land snails were found. Of that number, the majority were found on the island of Žirje, 31 species (on 25 sites altogether). In the literature, 6 species had been recorded (19\%). That clearly indicates the absence of papers with the systematic lists of land snail malacofauna that would result from any detailed field work of this island. All species mentioned in the literature were found in our field research. In the fauna of Zirje we emphasize the recently described Hypnophila zirjensis, found so far only on this island (ŠтамоL et al., 2018). It mostly resembles Hypnophila-species of the western Mediterranean joined in the subgenus Gomphroa (Šтамоl et al., 2018). In Croatia Hypnophila pupaeformis was known, distributed along the east Adriatic coast from the island of Pag in the north (FIscher et al., 2000) to Albania in the south (BANK, 2013). H. pupaeformis also inhabits Žirje, and on two localities $H$. pupaeformis and $H$. zirjensis live syntopically. Our research has shown that $H$. zirjensis inhabits habitats similar to $H$.

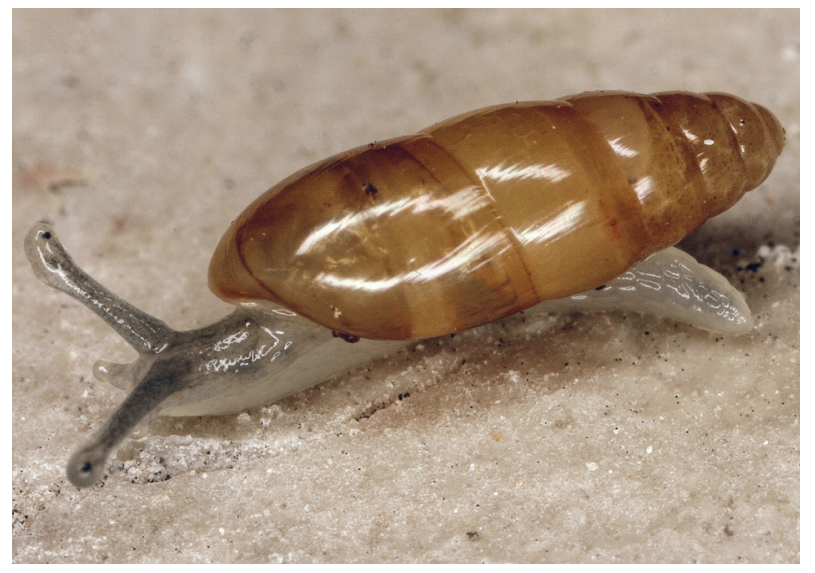

Fig. 3. Hypnophila zirjensis, snail found so far only on the island of Žirje (photo: H. Bilandžija, edited by B. Krstinić). 

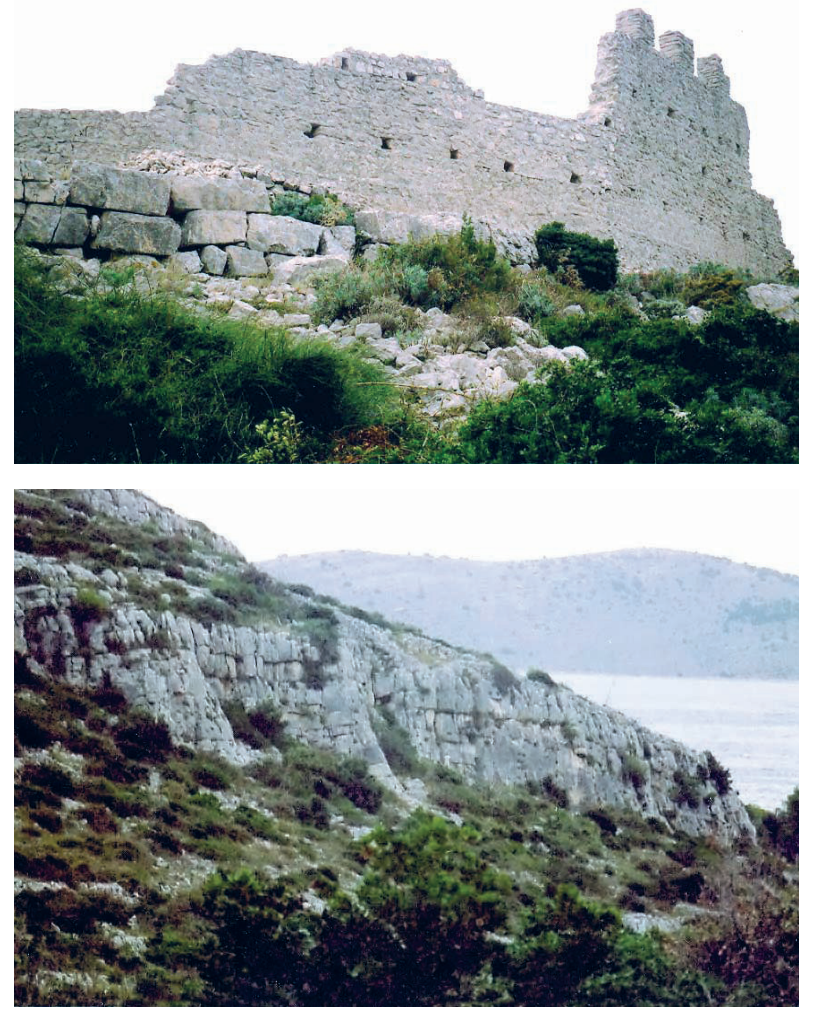

Fig. 4. Gradina locality on the island of Žirje, one of the finding sites of Hypnophila zirjensis (photo: V. Štamol).
Fig. 5. Luka bay on the island of Kaprije, a typical rocky habitat on the island (photo: V. Štamol).

pupaeformis: rock crevices, under the stones and speleological features, as was already known for H. pupaeformis (GitTenberger, 1993). During our research into the eastern Adriatic, live specimens of $H$. pupaeformis were almost always found in caves and pits, and something similar was noted by GitTEnberger (1993), which caused this species to be considered a troglophilic species (Gittenberger, 1993; GotTstein-MatočEc et al., 2001; ŠTAMOL, 2002), and we consider its placement into trogloxene taxa to be inaccurate (Vujčić-KARLO, 2008). We also found live H. zirjensis in a pit, and it definitely can spend its entire life cycle there; although considering its habitats on other sites, it can spend its life also outside pits, so we consider it a troglophile as well.

During field work on 17 sites on the island of Kaprije we found 27 species of land snails. In the available literature there were no taxa recorded for this island, which shows that there was either no research into its land malacofauna, or that there are no published results of such research. Among collected species we emphasize Truncatellina lussinensis, so far recorded only for the island of Lošinj (Croatia) (SтAмoL, 1995). According to the research of Massimo Prodan, morphological parameters of the Lošinj populations of $T$. lussinensis and of local T. callicratis do not overlap (Welter-Schultes, 2012). On Kaprije T. lussinensis was found at only one locality (2 specimens), syntopically - as on Lošinj with T. callicratis (6 specimens). There are too few specimens to allow of a valid statistical analysis of morphological characters of these taxa. The possible main reasons for doubting the validity of T. lussinensis are not the morphological parameters of T. lussinensis and T. callicratis. We think, as is emphasized in the description of T. lussinensis (ŠTAmoL, 
1995), that specimens of T. lussinensis could be abnormal, gigantic shells of the species T. callicratis. GEYER (1912) wrote about the phenomenon of gigantism, and most of abnormalities described for gigantic specimens of T. cylindrica (A. Férussac, 1807) were found with T. lussinensis. This thesis is in addition supported by the facts that both on Kaprije and on Lošinj specimens of T. lussinensis were found together with T. callicratis specimens, and that there were only few of T. lussinensis specimens as compared to the number of T. callicratis specimens. In any case modern methods, primarily DNA analysis, will give a correct answer. According to personal communication (De Mattia, 2016), specimens similar to T. lussinensis were also found in Istria (Brestova bay).

On the island of Prvić in the field work research at 9 localities we found 20 species of land snails. Only one of these taxa was recorded in the available literature, and that was over 160 years old (Strobel, 1854). This shows there has been either no systematic field research into its land malacofauna or no publication of any of the results of such research. In the fauna of Prvić we emphasize Delima (S.) vidovichii vidovichii, until now found only on the neighbouring mainland, which makes this its first island finding site.

Interesting results are species found only at one or at a few localities, especially if these localities are only on one of the researched islands. Species found only on Prvić are Chilostoma (L.) setosa setosa (1 locality) and Delima (S.) vidovichii vidovichii (2 localities). Species found only on Kaprije are Rumina decollata and Truncatellina lussinensis, both found only at one locality. Species found only on Žirje are Acanthinula aculeta (1 locality), Pseudochondrula seductilis seductilis, Granopupa granum (2 localities), and Hypnophila zirjensis (8 localities). The finding of Truncatellina lussinensis on one locality could be compared with same result on Lošinj, and it could be attributed to a general scarcity of this taxon. The distribution range of Delima (S.) vidovichii vidovichii could be the reason for its finding only on Prvić, because on Kaprije and Žirje it is replaced by Delima (S.) vidovichii robusta. That there are only two sites of Delima (S.) vidovichii vidovichii does not inevitably show its great scarcity considering the small number of research sites on Prvić in total. The explanation for the scarcity of other taxa could be attributed to the very few favourable habitats (e.g. forest habitats - Acanthinula aculeata, or grassland habitats - Pseudochondrula seductlis seductilis, Granopupa granum), or to very localized appearance (Rumina decollata, Chilostoma (L.) setosa setosa). We can also not exclude mistakes in the field work. The rare appearance could imply an endangered taxon, and favourable habitats on such localities should be protected. Another interesting result is the finding of the relatively frequent east Adriatic species on only one island, although at several sites. These are Monacha (Monacha) parumcincta found only on Žirje (21 out of 25 researched localities), and Aegopis acies found only on Prvić (4 out of 9 researched localities). It is difficult to find an explanation for this, since there are favourable habitats on other researched islands as well. The malacofauna of the researched islands was compared to the closest possible islands of a similar size. For Prvić $\left(2.41 \mathrm{~km}^{2}\right)$ this was Piškera, an island of the Kornati archipelago to the north $\left(2.68 \mathrm{~km}^{2}\right)$. On both islands the research included the same number of localities. On Piškera, which is a bit bigger, we found fewer species (14) (ŠтAмоL et al., 2014) than on Prvić (20 species). This could be the consequence of moderate anthropogenic influences on Prvić (conditions from 2008) which creates certain habitats lacking on the unihabited Piškera. Žirje $\left(15.08 \mathrm{~km}^{2}\right)$ was compared to the more northerly situated Silba $\left(14.28 \mathrm{~km}^{2}\right.$ Duplančić et al., 2004), where in the field work we found 23 species (ŠTAMOL, 2013). Although there were only half as many localities visited on Silba, we consider the lower habitat diversity to be the main reason of smaller number of species on Silba. 


\section{CONCLUSION}

During field research into three north Dalmatian islands of the Šibenik archipelago (Croatia) whose malacofauna was poorly known (until now 7 recorded species) we found 38 species of land snails with shells. The highest number was on the biggest island of Žirje (31 species), the smallest on the smallest island of Prvić (20 species), and on the middle-sized Kaprije there were 27 species. All seven species previously recorded in literature were confirmed by our research. The fauna is dominated by frequent and/or expected species for the eastern Adriatic or parts of it, such as Cochlostoma (Cochlostoma) scalarinum scalarinum, Pomatias elegans, Delima (Semirugata) semirugata semirugata, Hypnophila pupaeformis, Eobania vermiculata, Monacha (Monacha) cartusiana, Poiretia cornea. We emphasize interesting finds such as the recently described Hypnophila zirjensis with locus typicus and all its known finding sites on Zirje, which is so far endemic for this island; Truncatellina lussinensis, which on Kaprije has its second published finding site; and Delima (S.) vidovichii vidovichii, which on Prvić has its first island finding site. The researched islands were poorly inhabited, and the traditional economy (olive growing, vineyards, fishing, small crafts) with moderate and traditionally guided urbanization, created some new habitats and enabled a richer malacofauna than that existing on similar sized uninhabited eastern Adriatic islands. Unfortunately, island depopulation, especially on Kaprije and Žirje, with changes in the traditional function of an island as a permanent place for living, into places for holiday and recreation, with seasonal inhabitants, leading to urbanization of almost exclusively the coastal area where habitats are destroyed by concrete structures, and the abandonment of agriculture, which results in the arable land and meadows being taken over by forests of the Aleppo pine and Mediterranean macchia (FARIČIć \& MAGAš, 2004), inevitably will lead to lower diversity of habitats and to the reduction of the area of favourable habitats, which will endanger the islands' land snails. Local inhabitants of Žirje and Prvić recognized dangers of an uncontrolled tourism development and they are trying their best to withstand it, and those efforts along with nature protection should be supported by appropriate county and state institutions.

\section{ACKNOWLEDGEMENT}

We are thankful to senior museum taxidermist Zlatko Godec and retired senior museum technician Branko Jalžić for their help in the field work, and to Dr Helena Bilandžija for the snail photos. The research on Žirje and Kaprije was financed in part from the project of the Ministry of Science and Technology and former Ministry of Development and Reconstruction of the Republic of Croatia, and by the Croatian Natural History Museum, »Fauna of the Croatian Adriatic Islands« (No. 183005), and the research on Prvić by the COAST (a project of the Ministry of Environmental Protection, Spatial Planning and Construction of the Republic of Croatia with the support of UNDP in Croatia).

Received February 22, 2018

\section{REFERENCES}

BANK, R. A., (ed.), 2013: Mollusca, Gastropoda. Fauna Europaea version, 2.6.2, http://www.faunaeur.org/. BARIŠIĆ, T., 1994: Speleološka istraživanja otoka Žirja i Kornatskih otoka. [Speleological explorations of Žirje island and Kornati archipelago]. Žirajski libar 1, 183-193. [in Croatian, with English summary] 
BognaR, A. \& Saletto-Janković, M., 1994: Geomorfološke značajke otoka Žirja s arhipelagom. [Geomorphological characteristics of the island Žirje]. Žirajski libar 1, 169-182. [in Croatian, with English summary]

BRUSINA, S., 1872: Naravoslovne crtice sa sjevero-istočne obale jadranskoga mora, sabrao god. 1868 i 1871. Dio prvi. Rad JAZU 19, 105-177.

BuRšrć, I. (ed.), 2013: Census of population, households and dwellings 2011, population by sex and age. 1468 Statistical Reports. Croatian Bureau of Statistics, Zagreb, pp. 681.

Duplančić Leder, T., Ujević, T. \& ČAlA, M., 2004: Coastline lenghts and areas of islands in the Croatian part of the Adriatic sea determined from the topographic maps at the scale of $1: 25000$. Geoadria 9 (1), 5-32.

FARIČIĆ, J. \& MAGAš, D., 2004: Suvremeni socio-geografski problemi malih hrvatskih otoka - primjer otoka Žirja. Geoadria 9 (2), 125-158.

Fehér, Z., Deli T. \& Sólymos, P., 2010: Revision of Granaria frumentum (Draparnaud 1801) (Mollusca, Gastropoda, Chondrinidae) subspecies occurring in the eastern part of the species' range. J. Conchol. 40, 201-217.

Fischer, W., Kittel, K., Reischütz, A. \& Reischütz, P. L., 2000: Ein Beitrag zur Kenntnis der Molluskenfauna von Pag (Nord-Dalmatien, Kroatien). Nachrichtenbl. Ersten Vorarlberger Malak. Ges. 8, 53-59.

Franjić, J. \& PANDžA, M., 1996: Flora otoka Kaprija. Prirodna podloga, zaštita, društveno i gospodarsko valoriziranje Kornata. Ekološke monografije 7, 205-218.

Frank, C., 1991: Über Molluskenfunde aus Jugoslawien und den der Küste vorgelagerten Inseln des adriatischen Meeres. Zeitschr. angew. Zool. 78 (3), 349-380.

Friganović, M. A., 1994: Žirje - prilog poznavanju fiziogeografskih osobitosti [L 'ile de Žirje - contribution a la connaissance de ses caractères physiqus]. Zirajski libar 1, 62-72. [in Croatian, with French summary]

Geyer, D., 1912: Anomalie oder Artenbildung? Nachrichtsbl. deutsch. malakozool. Ges. 44 (3), 117-124.

Gittenberger, E., 1993: The Southeast European Hypnophila species (Mollusca: Gastropoda Pulmonata: Cochlicopidae). Zool. Meded. 67, 517-524.

Gottstein-Matočec, S. (ed.), Bakran-Petricioli, T., Bedek, J., Bukovec, D., Buzjak, S., Franičević, M., Jalžić, B., Kerovec, M., Kletečki, E., Kovačić, M., KralJ, J., Kružić, P., Kučinić, M., Kuhta, M., Matočec, N., Ozimec, R., RaĐA, T., Štamol, V., Ternjej, I. \& Tvrtković, N., 2001: Croatia. Pp. 22372287. In: Juberthie, C. \& V. Decu (eds.): Encyclopaedia Biospeologica. III. Société internationale de Biospéologie, Moulis - Bucarest.

JALŽić, B., 1994: Jama Gradina na otoku Žirju [The pit Gradina on Žirje island]. Žirajski libar 1, 45-47. [in Croatian, with English summary]

Milović, M. \& PANDŽA, M., 2010. A contribution to the vascular flora of the Šibenik archipelago islands (Dalmatia, Croatia). Nat. Croat. 19, 179-203.

PANDŽA, M., 1998: Flora of the islands of Krapanj and Prvić. Nat. Croat. 7, 321-339.

PANDŽA, M., 2003: Flora of the island of Žirje and the small islands around it (eastern Adriatic coast, Croatia). Acta Bot. Croat., 62 (2), 115-139.

Razkin, O., Gómez-Moliner, B. J., Vardinoyannis, K., Martínez-Ortí, A. \& Madeira, J. M., 2017: Species delimitation for cryptic species complexes: case study of Pyramidula (Gastropoda, Pulmonata). Zoologica Scripta 46 (1), 55-72.

Strobel, P., 1854: Molluschi terrestri raccolti da Cristoforo Bellotti nel 1853 in Dalmazia, con note ed aggiunte di P. Strobel. Giornale di Malacologia 2, 49-62, 114-123, 136-141, 145-151.

ŠTAMOL, V., 1986: Contribution to the study of land snails (Gastropoda) of the island of Brač and endargement of endemic species. Biosistematika 12 (1), 45-56. [in Croatian]

ŠтAмоL, V., 1995: Truncatellina velkovrhi $\mathrm{n}$. sp. and Truncatellina lussinensis n. sp., two new species from Croatia (Gastropoda: Pulmonata: Vertiginidae). Arch. Molluskenkunde 124 (1/2), 97-101.

ŠтамоL, V., 2002: 6.2. Hypogean terrestrial fauna. Invertebrates. Mollusca. Gastropoda. Pp. 44-47. In: Gotтstein Matočec, S. (ed..): An overview of the cave and interstitial biota of Croatia. Nat. Croat. 11, Suppl. 1, 1-112.

ŠTAMOL, V., 2004: Terrestrial snails (Mollusca: Gastropoda terrestria) of the Telašćica Nature Park (Dugi otok, Croatia). Nat. Croat. 13 (2), 95-113.

ŠтAмоL, V., 2010: A list of the land snails (Mollusca: Gastropoda) of Croatia, with recommendations for their Croatian names. Nat. Croat. 19 (1), 1-76. 
Štamol, V., 2013: Kopneni puževi (Gastropoda terrestria) otoka Silbe. Pp. 103-110. In: MužInIć, J. \& Purger, J. J. (eds.): Otok Silba, prirodno i kulturno blago. Sveučilište u Zadru, Zadar, 322 pp.

Štamol, V., Cameron, R., KletečKi, E., Vuković, M. \& Grgurev, M., 2014: Species/area and other relationships in land snails (Mollusca: Gastropoda terrestria) faunas of some adriatic islands. Nat. Croat. 23 (2), 317-334.

ŠtAmol, V., ERőss, Z. P., KLeTEČKI, E. \& VuKović, M., 2017: Terrestrial snails (Mollusca: Gastropoda) of islands of Šolta, Drvenik veli and Drvenik mali (Croatia). Nat. Croat. 26 (1), 45-64.

ŠtAmol, V. \& KLETEČKI, E., 2005: Terrestrial snails (Mollusca: Gastropoda terrestria) of Dugi otok (Croatia). Nat. Croat. 14 (1), 1-28.

ŠтAmol, V., KLeteČKI, E. \& Vuкоvić, M., 2012: A contribution to the knowledge of the terrestrial snails (Mollusca: Gastropoda terrestria) of Kornati National Park (Croatia). Nat. Croat. 21 (2), 427-454.

Štamol, V., Manganelli, G., Barbato, D. \& Giusti, F., 2018: Hypnophila zirjensis n.sp., a new azecid snail from the Croatian island of Žirje (Gastropoda: Pulmonata: Orthurethra). J. Conchol. 43 (1), 89-102.

Štamol, V. \& Poje, M., 1998: The fossil and recent malacofauna of the island of Susak (Croatia) (Gastropoda: Prososbranchia, Basommatophora, Stylommatophora). Malak. Abh. Mus. Tierkd. Dresden 19 (1), 103-117.

Štamol, V. \& Velкovrh, F., 1995: Contribution to the knowledge of land snail fauna of the islands of Cres and Lošinj (Croatia) (Gastropoda: Prosobranchia, Basommatophora, Stylommatophora). Malak. Abh. Mus. Tierkd. Dresden 17 (20), 219-237.

Topografske Karte [Topographic Maps], 1984: scale 1:25000, sheets Otok Žirje-istok (106-3-1), Otok Žirje-zapad (106-3-2), Vodice (106-4-1). Vojnogeogr. inst., Rep. geod. uprava SR Hrvatske, Zagreb.

Vujčić-Karlo, S., 2008: Skriveno mnoštvo endema. 115-151. In: BRmbota-Devčić, T., Lučić, I. (eds.): Krš bez granica. Pp. 157. Zbor novinara za okoliš Hrvatskog novinarskog društva, Zagreb, Centar za karstologiju ANUBiH, Sarajevo, Centar za krš i priobalje Sveučilišta u Zadru, Zagreb-Sarajevo.

Welter-Schultes, F. W, 2012: European non-marine molluscs, a guide for species identification. A1-A3, 679, Q1-Q78 pp. Planet Poster Editions, Göttingen.

Zallot, E., Groenenberg, D. S. J., De Mattia, W., Fehér, Z. \& Gittenberger, E., 2015: Genera, subgenera and species of the Cochlostomatidae (Gastropoda, Caenogastropoda, Cochlostomatidae). Basteria $78(4-6), 63-88$. 
